\title{
Efecto del fuego y dinámica de las hidrófitas emergentes en el humedal de la Mintzita, Michoacán, México
}

\author{
Fire and dynamics of the emergent hydrophytes of the Mintzita wetlands, Michoacán, México
}

\author{
Yazmín Escutia-Lara ${ }^{1}$, Sabina Lara-Cabrera ${ }^{1}$ y Roberto A. Lindig-Cisneros ${ }^{2 *}$ \\ ${ }^{1}$ Facultad de Biología, Universidad Michoacana de San Nicolás de Hidalgo, Morelia, Michoacán, México. \\ ${ }^{2}$ Laboratorio de Ecología de Restauración, Centro de Investigaciones en Ecosistemas, Universidad Nacional Autónoma de México. Apartado Postal \\ 27, Admón. 3, Santa María, 58091, Morelia, Michoacán, México. \\ *Correspondencia: rlindig@oikos.unam.mx
}

\begin{abstract}
Resumen. El estudio de la dinámica de humedales de aguas interiores de México, en particular del estado de Michoacán, ha sido muy limitado. Por lo tanto, en el año 2005 se inició un estudio de campo a través de parcelas permanentes para conocer la abundancia y distribución, a lo largo del tiempo, de las hidrófitas emergentes de los humedales de la Mintzita, en las cercanías de la ciudad de Morelia. A un año de iniciarse el trabajo (2006), la zona sufrió los efectos de un incendio que se propagó desde un terreno agrícola, disturbio que se repitió durante el tercer año del estudio (2007). Estos incendios permitieron documentar los efectos del fuego sobre la comunidad de hidrófitas emergentes en este sistema de humedales. Los efectos del fuego fueron diferenciales para las especies dominantes; Shoenoplectus americanus no fue afectada por el fuego, pero Typha domingensis redujo y modificó su cobertura a lo largo del gradiente hídrico, lo que ocasionó una pérdida de la zonación inicial característica de estas 2 especies. Durante el año 2005 se identificaron 34 especies; el siguiente año se perdieron 5, y finalmente en 2007 sólo se registraron 26. Entre las especies que se conservan, destaca Carex comosa, perteneciente a un género característico de humedales bien conservados, que aumentó su cobertura $133 \%$. Los incendios afectaron de manera más intensa a especies facultativas y pastos, lo que sugiere que disturbios semejantes pueden mantener la estructura y composición florística de este tipo de ecosistemas.
\end{abstract}

Palabras clave: humedal, dinámica, riqueza de especies, hidrófitas, fuego.

\begin{abstract}
Study of the dynamics of continental wetlands in México, and in particular from the state of Michoacán, has been scarce. In response to this need, a field study was started in 2005 through the establishment of permanent plots to document changes in abundance and distribution through time of emergent hydrophytes of the Mintzita wetlands, near the city of Morelia. In the first and third years, the area suffered the effects of a fire that propagated from an agricultural field. These fires allowed us to document the effects of fire on the emergent hydrophyte community in this wetland system. Fire effects were different for the different dominant species. Schoenoplectus americanus was not affected by fire but cover of Typha domingensis was reduced and modified across the wetland gradient, with the consequent loss of the zonation characteristic of these species. We identified 34 species during 2005; 5 species were not seen the following year, and in 2007 only 26 species were recorded. Among the species that persisted, Carex comosa, a species that belongs to a genus found in well preserved wetlands, stands out for having increased its cover $133 \%$. Fires had a severe effect on facultative species and grasses, suggesting that this type of disturbance can maintain the floristic composition and structure of these type of ecosystems.
\end{abstract}

Key words. wetland, dynamics, species richness, hydrophytes, fire.

\section{Introducción}

Los humedales se caracterizan por presentar condiciones de hipoxia al menos durante una parte del año, y esta propiedad es determinada por el régimen hidrológico, la que define la mayor parte de los atributos de las comunidades vegetales de los humedales así

Recibido: 11 abril 2008; aceptado: 24 febrero 2009 como de su dinámica (Cronk y Fennessy, 2001). Esto es particularmente notable en los humedales riparios y costeros, donde hay una zonación clara que sigue el nivel de inundación desde las partes más altas dominadas por especies que toleran periodos cortos de inundación, seguido por una zona dominada por hidrófitas emergentes y finalmente otra dominada por hidrófitas sumergidas. Este patrón se observa en los principales cuerpos de agua del estado de Michoacán: Cuitzeo, Pátzcuaro y Zirahuén 
(Rojas Moreno y Novelo Retana, 1995; Lot y Novelo, 1988; Madrigal Guridi et al., 2004), y también en otros cuerpos de agua de la región (Ramos Ventura y Novelo Retana, 1993) y del país (Bonilla-Barbosa y Novelo Retana, 1995). Sin embargo, otros tipos de disturbio pueden regular la composición de especies de las comunidades vegetales y los humedales no son la excepción.

Uno de los factores de disturbio más importantes es el fuego (una revisión reciente de sus efectos en zonas riparias es la de Pettit y Naiman, 2007). Los efectos del fuego en humedales se han estudiado tanto en humedales costeros como en los de aguas interiores, habiéndose obtenido una diversidad de respuestas que incluyen cambios en la composición de especies vegetales (Johnson y Knapp, 1995; Kost y De Steven, 2000), cambios en la composición e interacciones de las poblaciones de insectos (Hochkirchi y Adorf, 2007), los efectos sobre poblaciones de aves acuáticas (Gabrey et al., 1999, Brennan et al., 2005), en la dinámica de nutrientes (Laubhan, 1995) y en la producción primaria (Gabrey yAfton, 2001).

En algunos tipos de humedales, los incendios son parte de la dinámica natural de disturbio y hay especies hidrófitas adaptadas a estas condiciones; por ejemplo, Cladium jamaicense cuyas hojas se secan en invierno y son muy inflamables, pero posee rizomas sumergidos que retoñan rápidamente después de un incendio, pudiendo crecer hasta $40 \mathrm{~cm}$ en 2 semanas (Kushlan, 1990). Los efectos del fuego son variables y dependen del tipo de humedal. Por razones de manejo, en zonas donde el fuego fue suprimido pero es parte de la dinámica natural es donde más se han estudiado sus efectos (Kost y De Steven, 2000). Por ejemplo, en humedales dominados por especies del género Carex en la parte norte de los Estados Unidos, el fuego favorece a las especies anuales (Warners, 1989). Sin embargo, en marjales de la misma área geográfica, que son un tipo de humedales relacionados con el anterior pero caracterizados por estar alimentados de agua rica en calcio y magnesio, los incendios favorecen a las gramíneas y reducen la diversidad de especies anuales (Bowles et al., 1996), lo que sugiere que las respuestas al fuego de los distintos tipos de comunidades de hidrófitas emergentes en los humedales dependen de la composición florística específica de cada tipo de humedal y de su interacción con factores abióticos.

Existen estudios sobre la composición florística y otros aspectos de la vegetación de diversos humedales en México, que incluyen humedales de cuerpos de agua interiores (Rojas Moreno y Novelo Retana 1995, Lot y Novelo 1988, Madrigal Guridi et al. 2004) así como de ambientes marinos (Collado Vides et al. 1995, Zedler et al. 1999, Romero López et al. 2006, Echavarria-Heras et al. 2007). Sin embargo, en una búsqueda realizada en los principales índices de 1965 a la fecha (ISI-Web of Knowledge, Biological Abstracts, Agricola y, para revistas iberoamericanas, en REDALYC), no se encontraron registros de artículos que traten sobre los efectos del fuego en humedales de aguas interiores de México.

Como parte de un trabajo que incluye aspectos de dinámica de nutrientes (Escutia-Lara et al., 2008) y de diversos factores de disturbio (Hall et al., 2008), para conservar y restaurar los humedales asociados a los manantiales de la Mintzita, en 2005 se inició un estudio a largo plazo en su parte mejor conservada, con niveles relativamente bajos de disturbio antrópico y ningún incendio, al menos desde inicios de la década (EscutiaLara, obs. pers.).

Los manantiales de la Mintzita y sus humedales, a pesar de contar con una extensión menor que los de Pátzcuaro y Cuitzeo -los mejor conocidos del estado de Michoacán-, son refugios de fauna y flora, así como abastecedores de agua para la ciudad de Morelia (capital del estado a la que aportan aproximadamente $40 \%$ del agua potable). Los incendios provocados en 2006 y 2007 permitieron evaluar el efecto de este tipo de disturbio en la composición de la comunidad de hidrófitas emergentes.

\section{Materiales y métodos}

Área de estudio. Los manantiales de la Mintzita que se encuentran a $7 \mathrm{~km}$ al sur de la ciudad de Morelia, Michoacán, se localizan a una altitud de $1917 \mathrm{~m} \mathrm{snm}$, en el municipio de Morelia, Michoacán. Se encuentran a sólo $4.5 \mathrm{~km}$ al sur de la carretera federal - 15 por la rama estatal a Cointzio-, a $7.5 \mathrm{~m}$ en línea recta al noroeste del poblado de Cointzio y 500 metros al suroeste $\left(101^{\circ} 17^{\prime} 42^{\prime \prime}\right.$ O y $\left.19^{\circ} 38^{\prime} 43^{\prime \prime} \mathrm{N}\right)$ (INEGI, 1998). En la región se presenta un clima templado subhúmedo, $\mathrm{Cb}(\mathrm{w})(\mathrm{w}) \mathrm{i}^{\prime} \mathrm{g}$, con lluvias en verano y un coeficiente de P/T entre 55 y 43.2, con lluvias invernales que representan menos del $5 \%$ de la precipitación pluvial; poca oscilación térmica, con marcha de la temperatura tipo Ganges (13 a $34{ }^{\circ} \mathrm{C}$; García, 1988). La vegetación terrestre circundante está constituida principalmente por matorral subtropical, en tanto que la acuática está formada por vegetación sumergida, Nymphaea mexicana, Stuckenia pectinata (syn. Potamogeton pectinatum) y Ceratophylum demersum; flotante, Eichhornia crassipes y arraigada emergente, la que es el foco de atención del presente estudio, en la que dominan en cobertura Typha domingensis y Schoneoplectus americanus (Rodríguez y Guevara, 2000).

Muestreo. Los humedales de la Mintzita son sujetos a diversos disturbios de origen antrópico; destacan el sobrepastoreo y la extracción de hojas de las especies 
dominantes, que son utilizadas como forraje, lo que ha generado un mosaico en la vegetación, en donde se pueden apreciar diversos grados de conservación. En los humedales del área que se aprecia como mejor conservada, pues no fue sometida a pastoreo en los 6 años anteriores al estudio (Escutia-Lara, obs. per.), se ubicaron 5 transectos perpendiculares a la orilla del cuerpo de agua con distancia de $30 \mathrm{~m}$ entre cada uno, iniciándose cada transecto en el borde de la vegetación hidrófita emergente y terminando donde el agua tenía $50 \mathrm{~cm}$ de profundidad. En esta última zona se encontraban exclusivamente individuos de Typha domingensis. A lo largo de cada transecto se ubicaron 6 parcelas equidistantes, cada una con un área de $1 \mathrm{~m}^{2}$. De cada parcela se registraron todas las especies presentes, sus coberturas, dividiendo la parcela en 100 partes iguales de $1 \mathrm{~m}^{2}$. De las zonas cercanas se colectaron ejemplares para su herborización y posterior identificación. Los muestreos se realizaron en el periodo de secas (mayo) y de lluvias (septiembre) de 2005-2007. Este método fue modificado del desarrollado por Great lakes environmental indicators project que permite un muestreo eficiente de este tipo de comunidades y que por tratarse de un método estandarizado permite comparaciones entre sitios (Frieswyk y Zedler, 2006, Frieswyk y Zedler, 2007, Frieswyk et al., 2007). Los datos de cobertura de cada especie se promediaron por año y se presentan como porcentaje de cobertura.

Para la identificación de las especies se emplearon las publicaciones de Lot (2000); Lot y Novelo (2004), Lot et al. (1998, 1999), Villaseñor y Espinosa (1998) y se corroboró la identidad de algunas especies gracias a la colaboración del Herbario de la Universidad de WisconsinMadison (WIS), donde se depositaron los ejemplares correspondientes. Un juego completo de los ejemplares se depositó en el herbario del Insituto de Ecología, en Pátzcuaro (IEB). No fue posible la determinación de 5 especies de la familia Poaceae, que a pesar de presentarse unos cuantos individuos de estos taxa en los 2 años de muestreo, no sobrevivieron más allá de la etapa de plántula o estadios muy tempranos, por lo que no fue posible su identificación. Para determinar el tipo de hábitat de las especies encontradas, adicionalmente se consultó Plants database (USDA, 2008), donde las plantas presentes en el humedal se clasifican como facultativas o estrictas en función de su presencia obligada o no en suelos hídricos. Para las especies de este estudio se corroboró que las clasificadas como facultativas se encontraban también fuera del humedal.

En el mes febrero de 2006 y de 2007, se presentaron incendios inducidos que se inciaron en parcelas agrícolas. Estos incendios abarcaron toda el área de estudio y aunque no es posible determinar su intensidad en términos de temperatura máxima alcanzada, el hecho de que consumieran toda la biomasa aérea seca de las plantas del humedal pero sin matar a los individuos de Salix bonplandiana, los cuales conservaron las ramas y parte del follaje, indica que no fueron muy intensos en términos de temperatura ni en duración, pues de otra manera, el tejido de esta especie leñosa.hubiera muerto.

Análisis estadístico. Para analizar los cambios a lo largo del tiempo en la cobertura de las especies dominantes que forman la matriz del humedal, $S$. americanus y $T$. domingensis, se plantearon 2 hipótesis estadísticas; la primera sobre los cambios en cobertura por especie y la segunda sobre la variación en la cobertura también por especie. Para probar la hipótesis de que los incendios afectaron la cobertura de las especies dominantes, se llevó a cabo un análisis por medio del método bootstrap, utilizando como variable el porcentaje de cobertura por parcela de cada especie, realizando 10000 muestreos con reemplazo de los valores de cobertura del primer año de muestreo, obteniendo el promedio de cobertura para todas las parcelas y comparando con el valor promedio de todas las parcelas del último año de muestreo (Crawley, 2007). De esta manera, se puede detectar si el valor promedio de cobertura después de los incendios corresponde a lo esperado por cambios al azar o no. Para determinar si existen diferencias en la variabilidad de la cobertura en las parcelas, se utilizó la prueba de varianzas de Fisher (Zar, 1999). Para detectar el patrón de la cobertura por especie dominante a lo largo del gradiente hídrico se utilizaron análisis de regresión. Los análisis antes descritos se llevaron a cabo con el paquete R ( R Development core team, 2008). Para analizar el cambio en riqueza de especies se utilizó el método de rarefacción (esfuerzo de muestreo/ riqueza de especies calculada); para comparar la riqueza antes y después de los incendios (Colwell et al., 2004), por medio del paquete EstimateS (Colwell, 2005). El estimador Chao1 se utilizó para determinar si el esfuerzo de muestreo en campo fue el adecuado (Chao et al., 2005). Un análisis de agrupamiento (cluster) se llevó a cabo para comparar la composición de especies entre los 3 años de estudio (Manly, 2000).

\section{Resultados}

Riqueza y composición de especies. En las parcelas permanentes se encontró un total de 34 especies en el año 2005, antes de los incendios. Para el año 2006, después del primer incendio se encontraron 29 especies y para el 2007, después del segundo incendio, 26 especies (Cuadro 1). La diferencia en la riqueza de especies es significativa si se comparan las curvas de rarefacción para los años 2005 y 2007 (Fig. 1), pues los intervalos de confianza no 
Cuadro 1. Especies presentes en las parcelas de los humedales de la Mintzia, Michoacán de 2005-2007. Porcentaje de cobertura anual, respuesta de cada especie y tipo de hábitat al que pertenecen

\begin{tabular}{|c|c|c|c|c|c|}
\hline Especie & 2005 & 2006 & 2007 & Respuesta & Hábitat \\
\hline Arenaria paludicota B.L. Robins & 3 & 1 & 1 & $\mathrm{R}$ & $\mathrm{H}$ \\
\hline Berula erecta (Huds.) Couille & 5 & 3 & 1 & $\mathrm{R}$ & $\mathrm{H}$ \\
\hline Bidens aurea (Ait.) Sherff & 1 & 0 & 0 & $\mathrm{P}$ & $\mathrm{F}$ \\
\hline Bidens cernua $\mathrm{L}$. & 2 & 1 & 1 & $\mathrm{R}$ & $\mathrm{H}$ \\
\hline Carex comosa Boott & 3 & 4 & 7 & A & $\mathrm{H}$ \\
\hline Cyperus eragrostis Lam. & 1 & 0 & 0 & $\mathrm{P}$ & $\mathrm{F}$ \\
\hline Cyperus niger $\mathrm{R}$ et $\mathrm{P}$. & 1 & 0 & 0 & $\mathrm{P}$ & $\mathrm{F}$ \\
\hline Dirsia sp. & 1 & 1 & 1 & $\mathrm{~S} / \mathrm{C}$ & - \\
\hline Eichhornia crassipes (C. Martius) Sol Ms-Laub. & 1 & 0 & 0 & $\mathrm{P}$ & $\mathrm{H}$ \\
\hline Eleocharis montevidensis Kunth & 1 & 3 & 2 & A & $\mathrm{F}$ \\
\hline Epilobium ciliatum Raf. & 1 & 0 & 0 & $\mathrm{P}$ & $\mathrm{F}$ \\
\hline Eupatorium rugosum Houtt. & 6 & 5 & 7 & A & $\mathrm{F}$ \\
\hline Galium trifidum $\mathrm{L}$. & 1 & 2 & 1 & A & $\mathrm{F}$ \\
\hline Gnaphalium americanum (P. Mill.) Wedell & 1 & 0 & 1 & $\mathrm{~S} / \mathrm{C}$ & $\mathrm{F}$ \\
\hline Hydrocotyle ranunculoides L. f. & 1 & 1 & 1 & $\mathrm{~S} / \mathrm{C}$ & $\mathrm{H}$ \\
\hline Hydrocotyle verticillata Thunb. & 8 & 8 & 8 & $\mathrm{~S} / \mathrm{C}$ & $\mathrm{H}$ \\
\hline Lobelia sp. & 1 & 1 & 0 & $\mathrm{P}$ & - \\
\hline Lythrum vulneraria $\mathrm{L}$. & 1 & 1 & 2 & A & $\mathrm{H}$ \\
\hline Mimulus glabratus Kunth & 2 & 6 & 7 & A & $\mathrm{H}$ \\
\hline Panicum sucosum Hitch. et Chase & 1 & 1 & 0 & $\mathrm{P}$ & $\mathrm{F}$ \\
\hline Phragmatis australis (Cav.) Trin. et Steud. & 3 & 4 & 4 & A & $\mathrm{H}$ \\
\hline Poaceae 1 & 3 & 2 & 2 & $\mathrm{R}$ & - \\
\hline Poaceae 2 & 1 & 1 & 1 & $\mathrm{~S} / \mathrm{C}$ & - \\
\hline Poaceae 3 & 1 & 0 & 1 & $\mathrm{~S} / \mathrm{C}$ & - \\
\hline Poaceae 4 & 1 & 0 & 1 & $\mathrm{~S} / \mathrm{C}$ & - \\
\hline Poaceae 5 & 2 & 1 & 0 & $\mathrm{P}$ & - \\
\hline Polygonum hydropiperoides Michx. & 1 & 1 & 2 & A & $\mathrm{H}$ \\
\hline Polygonum lapathifolium L. & 1 & 1 & 3 & A & $\mathrm{H}$ \\
\hline Rorippa palustris (L.) Besser & 1 & 1 & 0 & $\mathrm{R}$ & $\mathrm{H}$ \\
\hline Salix bonplandiana Kunth & 4 & 1 & 1 & $\mathrm{R}$ & $\mathrm{H}$ \\
\hline Schoenoplectus americanus (Pers.) Volkart ex Schinz et Keller & 18 & 21 & 16 & $\mathrm{R}$ & $\mathrm{H}$ \\
\hline Schoenoplectus tabernaemontani (C.C. Gmeling) Palla & 1 & 1 & 1 & $\mathrm{~S} / \mathrm{C}$ & $\mathrm{H}$ \\
\hline Solanum americanum P. Mill. & 1 & 0 & 1 & $\mathrm{~S} / \mathrm{C}$ & $\mathrm{F}$ \\
\hline Typha domingensis Presl. & 44 & 29 & 32 & $\mathrm{R}$ & $\mathrm{H}$ \\
\hline Número total de especies & 34 & 29 & 26 & & \\
\hline
\end{tabular}

Respuesta. $\mathrm{A}=$ aumentó su cobertura, $\mathrm{P}=$ especie que se pierde después de los incendios, $\mathrm{R}=$ reduce su cobertura, y $\mathrm{S} / \mathrm{C}=\sin$ cambio. Hábitat. $\mathrm{H}=$ especies estrictas del humedal, $\mathrm{F}=$ facultativas.

se sobrelapan; para el año 2005, la riqueza calculada es de $34 \pm 2.7$ especies y para 2007 , de $26 \pm 5.1$ especies, lo que significa que con un intervalo de confianza del 95\%, el valor mínimo para 2005 es de 31.3 especies y el máximo para 2007 es de 31.1 especies. Adicionalmente, el estimador Chao1 indica que en los 2 años que se comparan el muestreo fue adecuado. Para 2005 este indicador tiene un valor de 35.7 , que se encuentra dentro del intervalo de confianza para ese año, y para 2007 coincide con el número de especies encontradas (26). En términos de la composición de especies, el análisis de agrupamiento (cluster) indica que la composición de especies durante el 2005 es diferente a los 2 años posteriores (Fig. 2).

Del total de las especies que se encontraron, comparando entre el primer año antes de los incendios y el último año, 9 aumentaron su cobertura después de los 


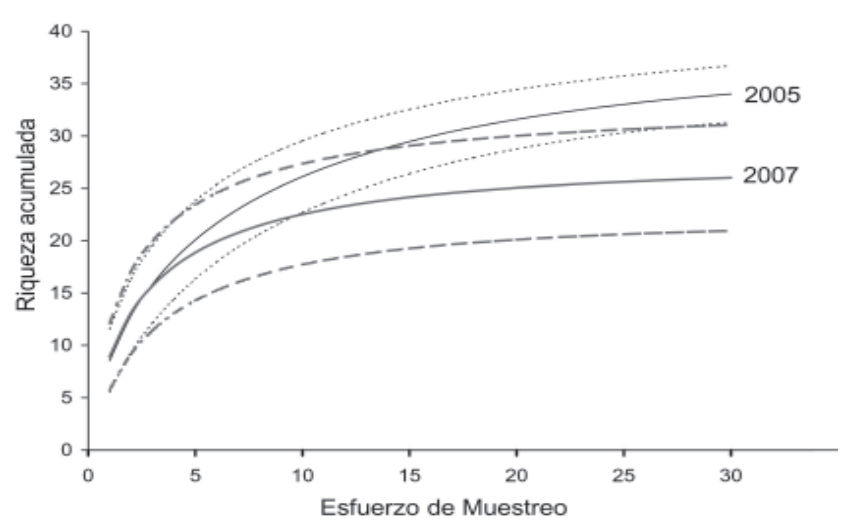

Figura 1. Curvas de rarefacción para la riqueza de especies en el año 2005, antes de los incendios, y en el año 2007, después del segundo incendio. La diferencia en la riqueza de especies entre ambos muestreos es significativa.

incendios (Cuadro 1). Las que destacan por presentar el mayor porcentaje de cambio son Carex comosa (133\% de incremento) y Mimulus glabratus (250\% de incremento). Del resto, 8 especies redujeron su cobertura, 9 no mostraron cambios y 8 desaparecieron de las parcelas permanentes.

De las 34 especies recoletadas en las parcelas permanentes, 10 se pudieron clasificar como especies facultativas, 17 como estrictas, 7 no se agruparon debido a que en 2 casos no fue posible identificar la especie (Dirsia sp. y Lobelia sp.) y 5 pastos no se desarrollaron más allá de las primeras etapas de vida. Entre las especies estrictas, 6 aumentaron su cobertura, del primer año antes de los incendios al tercero (35\%), y entre las especies facultativas, 3 aumentaron su cobertura (30\%). De las 5 especies de Poaceae que no se pudieron identificar, 3 mantuvieron su cobertura, una la redujo y la última se perdió de las parcelas permanentes.

Especies dominantes. A lo largo de los 3 años del estudio se detectaron diferencias en la cobertura de $S$. americanus y $T$. domingensis en las parcelas permanentes (Fig. 3). La cobertura de cada especie durante el primer año del estudio (Fig. 4) muestra el patrón esperado para las 2 especies dominantes, siendo $S$. americanus más abundante en las partes más cercanas a la orilla del humedal y T. domingensis en las partes más profundas. La variación en cobertura siguiendo el gradiente hídrico del humedal es significativo para ambas especies $\left(S\right.$. americanus: $\mathrm{R}^{2}=0.68$,

$\mathrm{F}_{(1,28)}=59.5, P<0.00001 ; T$. domingensis: $\mathrm{R}^{2}=0.67$, $\left.\mathrm{F}_{(1,28)}=57.6, \quad P<0.00001\right)$. En los 2 años posteriores (Fig 1, 2006 y 2007), este patrón se hace menos marcado, particularmente para el tercer año, cuando los valores promedio de cobertura (Fig. 1) y la varianza de los mismos cambia para ambas especies. Un ajuste de regresión para la cobertura de $T$. domingensis con respecto a la distancia
UPGMA
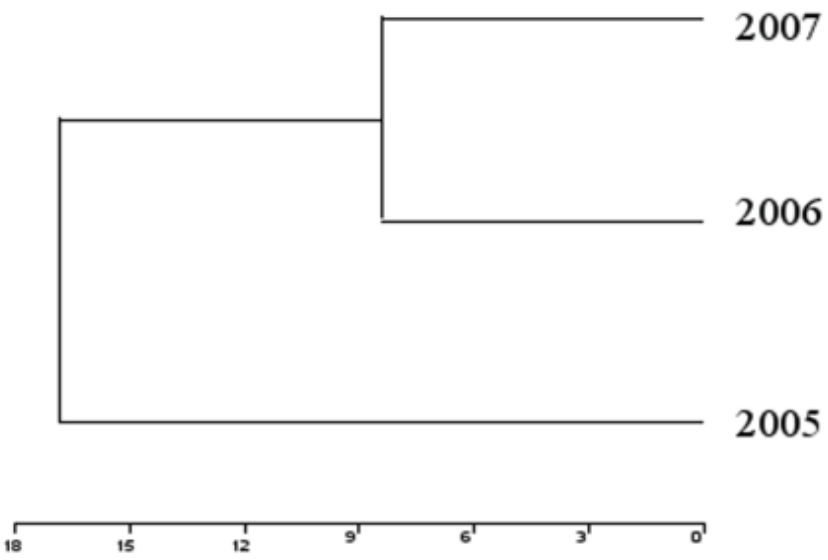

Figura 2. Dendograma del análisis de agrupamiento para las comunidades de plantas en los 3 años de muestreo. Las comunidades en los años 2006 y 2007 son muy similares entre sí, y ambas son diferentes de la de 2005.

a la orilla del humedal indica que el patrón es menos significativo que 2 años antes $\left(\mathrm{R}^{2}=0.29, \mathrm{~F}_{(1,28)}=11.6\right.$, $P=0.002$ ) con una reducción de $45 \%$ en el valor de $\mathrm{R}^{2}$, mientras que para $S$. americanus se mantiene sin cambios.

El análisis de bootstrap para $S$. americanus del cambio en cobertura no es estadísticamente significativo $(P=$ 0.39 ), a pesar de que la cobertura promedio de las parcelas cambió de $17.5 \%$ a $15.9 \%$ entre el año de 2005 y el año de 2007; de manera similar, el cambio en la varianza de las coberturas (246 en 2005 y 299 en 2007) tampoco es significativo $(P=0.61)$. Para $T$. domingensis, el cambio en cobertura (49\% en 2005 y $32 \%$ en 2007 ) fue significativo $(P<0.001)$ y también lo fue el cambio en la varianza de las coberturas (900 en 2005 y 351 en 2007, $P=0.013$ ), para esta especie hubo una reducción de cobertura del $17 \%$ y una reducción del $64 \%$ en la varianza de las coberturas

\section{Discusión}

Los resultados del seguimiento de la dinámica de la vegetación hidrófita emergente en las parcelas permanentes establecidas en los humedales del manantial de la Mintzita, permitieron documentar los efectos del fuego en la estructura y composición del humedal. Como en muchos humedales, la estructura de la vegetación está definida por pocas especies dominantes; en el caso de los de la Mintzita, por Typha domingensis y Shoenoplectus americanus. La estructura de la vegetación del humedal cambió después de los incendios debido a que $T$. domingensis redujo su cobertura en las zonas en las que 


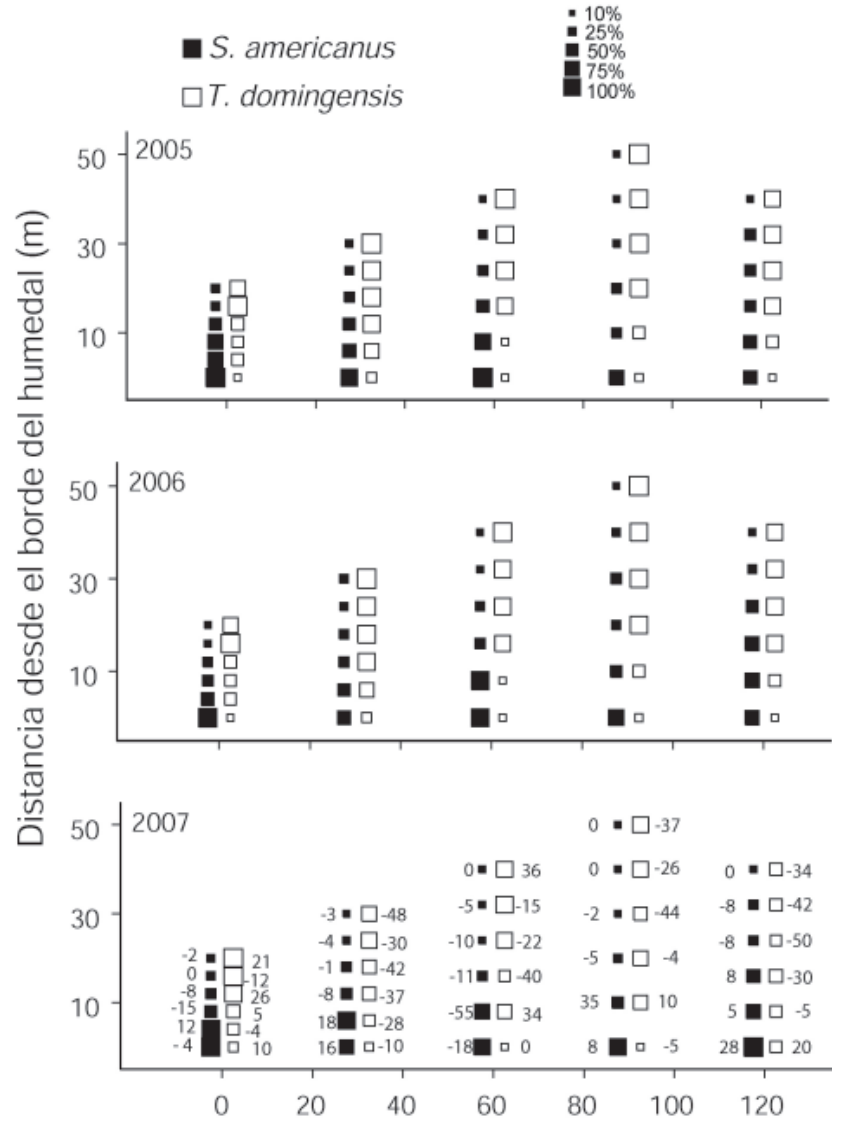

Distancia desde el extremo sur $(\mathrm{m})$

Figura 3. Cobertura de las especies dominantes en las parcelas permanentes de acuerdo con su posición en el humedal. Se grafica a partir del inicio de la vegetación hidrófita emergente. la longitud de cada transecto varía de acuerdo al gradiente hídrico del humedal.

era dominante, es decir, en las partes más profundas, y aumentó ligeramente su cobertura en las partes menos profundas, donde $S$. americanus dominaba antes de los incendios. Sorpresivamente, $S$. americanus no sufrió cambios significativos a lo largo de los 3 años del estudio. El efecto de los incendios observado en $T$. domingensis en otros estudios (Lee et al., 2005, Kostecke et al., 2004) es similar al de las parcelas de la Mintzita y consiste en una reducción de su cobertura, la cual no se recupera sino hasta varios años después del disturbio.

Cabe resaltar que la presencia de Carex comosa en las parcelas permanentes sugiere que los humedales de la Mintzita no han sufrido disturbios severos en el área estudiada; las especies de este género son características de sistemas bien conservados sujetos a patrones naturales de disturbio que pueden incluir dinámica de fuego

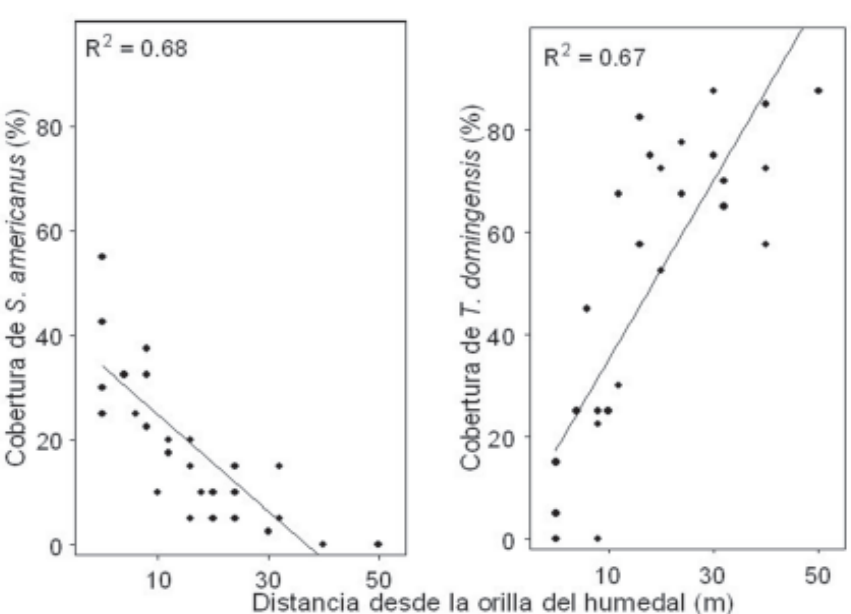

Figura 4. Patrones en la cobertura de las 2 especies dominantes en función del gradiente hídrico del humedal. De acuerdo con lo esperado, S. americanus disminuye su cobertura en medida que aumenta la distancia, y por lo tanto la profundidad, $T$ domingesis sigue el patrón inverso.

(Werner y Zedler, 2002). Además, el aumento en la cobertura de C. comosa después de los incendios sugiere un papel importante de estos en mantener a las especies más sensibles en la comunidad vegetal, porque reduce la cobertura de las dominantes. Se ha demostrado que la reducción de la cobertura de las especies dominantes reduce la competencia y favorece una mayor diversidad en este sistema (Hall et al., 2008). Al respecto, debe destacarse que después de los incendios las especies que menos pérdidas sufrieron fueron las estrictas del humedal, seguidas por las facultativas y finalmente por los pastos, lo cual sugiere que los incendios favorecen en general a las especies características del humedal. Si a lo anterior sumamos que también fue negativo el efecto de los incendios sobre la única especie leñosa presente (Salix bonplandiana), se puede plantear la hipótesis -que requeriría de estudios experimentales controlados- de que los incendios son benéficos para este tipo de humedales de aguas interiores en México. A esta hipótesis la avala el hecho de que el fuego tiene efectos similares sobre la composición de especies en humedales dominados por diversas especies de Carex (Kost y De Steven, 2000). En zonas de los manantiales de la Mintzita fuera del área de estudio y que no han sufrido el efecto del fuego en las últimas décadas, la vegetación está completamente dominada por T. domingensis, Phragmites australis y $S$. bonplandiana, lo que también apoya la hipótesis de que los incendios son positivos para este tipo de humedales.

Estos resultados representan un "experimento natural" debido a que permiten estudiar el efecto de un factor no 
anticipado sobre la dinámica del sistema o de efectos difíciles de manipular de manera experimental a la escala natural en la que ocurren; sin embargo adolecen de limitaciones, entre las que destacan la falta de controles apropiados o de réplicas (Hulbert, 1984). A pesar de ello, su valor para indicar patrones y posibles relaciones causales no debe subestimarse, sobre todo si se considera que a ciertas escalas las limitaciones mencionadas son inevitables, particularmente en sistemas muy heterogéneos (Hargrove y Pickering, 1992).

Sin ignorar lo anterior, los patrones observados en el presente estudio permiten proponer diversos aspectos sobre los humedales de aguas interiores de México que merecen ser considerados en estudios futuros; entre otros, el papel potencial del fuego como parte de la dinámica que permite mantener la identidad de las comunidades de hidrófitas emergentes. La respuesta diferencial de las especies estrictas y facultativas al fuego. El uso del fuego como una herramienta de manejo para este tipo de humedales, pues los resultados de este estudio sugieren que favorece a las especies características de los humedales.

\section{Agradecimientos}

A Steven Hall, por su ayuda en corroborar la identidad de algunas especies. A la Universidad de Wisconsin por el financiamiento otorgado por medio de Alumni Research Foundation, gracias al donativo del señor Ed Weigner y a la Universidad Nacional Autónoma de México, por el recibido del macroproyecto "Manejo de Ecosistemas y Desarrollo Humano” (SDEI-PTID-02).

\section{Literatura citada}

Bonilla-Barbosa, J. R. y A. Novelo R. 1995. Manual de identificación de plantas acuáticas del Parque Nacional Lagunas de Zempoala, México.Cuadernos del. Instituto de Biología, 26, Universidad Nacional Autónoma de México, México, D. F. 168 p.

Bowles, M., J. McBride, N. Stoynoff y K. Johnson. 1996. Temporal changes in vegetation composition and structure in a fire-managed prairie fen. Natural Areas Journal 16:275288.

Brennan, E. K., L. M. Smith, D. A. Hauberks y T. G. LaGrange. 2005. Short-term response of wetland birds to prescribed burning in rainwater basin wetlands. Wetlands 25:667674.

Chao, A., R. L. Chazdon, R. K. Colwell y T. Shen. 2004. A new statistical approach for assessing similarity of species composition with incidence and abundance data. Ecology Letters 8:148-159.

Collado-Vides, L., J. González González y E. Ezcurra. 1995. patrones de distribución ficoflorística en el sistema lagunar de Nichupté, Quintana Roo, México. Acta Botanica Mexicana 31:19-32.

Colwell, R. K. 2005. EstimateS: Statistical estimation of species richness and shared species from samples. Version 7.5. Persistent URL: <purl.oclc.org/estimates >.

Colwell, R.K., C. X. Mao y J. Chang. 2004. Interpolating, extrapolating, and comparing incidence-based species accumulation curves. Ecology 85:2717-2727.

Crawley, M. J. 2007. The R book, primera edición. Wiley, London. 492 p.

Cronk, J. K. y M. S. Fennessy. 2001. Wetland plants: biology and ecology. Lewis. Nueva York. 445 p.

Echavarria-Heras, H., E. Solana-Arellano y V. Díaz-Castaneda. 2007. Allometric scaling of dry weight and leaf area for Spartina foliosa in Punta Banda Estuary, Baja California, Mexico. Wetlands 27:270-277.

Escutia-Lara, Y., M. Gómez-Romero y R. Lindig-Cisneros. 2008. Nitrogen and phosphorus effect on Typha domingensis Presl. rhizome growth in a matrix of Schoenoplectus americanus (Pers.) Volkart ex Schinz and Keller. Aquatic Botany. doi:10.1016/j.aquabot.2008.06.002.

Frieswyk, C. B. y J. B. Zedler. 2006. Do seed banks confer resilience to coastal wetlands invaded by Typha $\mathrm{x}$ glauca? Canadian Journal of Botany 84:1882-1893.

Frieswyk, C. B. y J. B. Zedler. 2007. Vegetation change in Great Lakes coastal wetlands: Deviation from the historical cycle. Journal of Great Lakes Research 33:366-380.

Frieswyk, C. B., C. Johnston y J. B. Zedler. 2007. Quantifying and qualifying dominance in vegetation Journal of Great Lakes Research 33(Special Issue 3):125-135.

Gabrey, S. W. y A. D. Afton. 2001. Plant community composition and biomass in Gulf Coast Chenier Plain marshes: Responses to winter burning and structural marsh management Environmental Management 27:281-293.

Gabrey, S. W., A. D. Afton y B. C. Wilson. 1999. Effects of winter burning and structural marsh management on vegetation and winter bird abundance in the Gulf Coast Chenier Plain, USAWetlands 19:593-606.

García, E. 1988. Modificaciones al sistema de clasificación climática de Köppen, $4^{\mathrm{a}}$ edición, CETENAL, México, D. F. $213 \mathrm{p}$.

Hall, S., J.B. C. Zedler y R. Lindig-Cisneros. 2008. Does harvesting sustain diversity in central Mexican wetlands? Wetlands 28:776-792.

Hargrove, W. W. y J. Pickering. 1992. Pseudoreplication: a sine qua non for regional ecology. Landscape Ecology 6:251258.

Hochkirchi, A. y F. Adorf. 2007. Effects of prescribed burning and wildfires on Orthoptera in Central European peat bogs. Environmental Conservation 34:225-235.

Hulbert, S. H. 1984. Pseudoreplication and the design of ecological field experiments. Ecological monographs 54:187-211.

INEGI (Instituto Nacional de Estadística, Geografía e Informática). 1998. Carta topográfica Morelia, E14-1. Escala 1:250,000. Michoacán, México. INEGI, Aguascalientes, Aguascalientes.

Johnson, S. R. y A. K. Knapp. 1995. The influence of fire on 
Spartina pectinata wetland communities in a northeastern Kansas tallgrass prairie. Canadian Journal of Botany 73:8490.

Kost, M. A. y D. Steven. 2001. Plant community responses to prescribed burning in Wisconsin sedge meadows. Natural Areas Journal 20673-687:36-45.

Kostecke, R. M., L. M. Smith y H. M. Hands. 2004. Vegetation response to cattail management at Cheyenne Bottoms, Kansas. Journal of Aquatic Plant Management 42:39-45.

Kushlan, J.A. 1990. Freshwater marshes. In Ecosystems of Florida, R.L. Myers y J.J. Ewel, (eds.), University of Central Florida Press, Orlando. p. 324-363.

Laubhan, M. K. 1995. Effects of prescribed fire on moist-soil vegetation and soil macronutrients. Wetlands 15: 159-166.

Lee, M. A. B. , K. J. Ponzio y S. J. Miller. 2005. Response of willow (Salix caroliniana Michx.) in a floodplain marsh to a growing season prescribed fire. Natural Areas Journal 25:239-245.

Lot, A. y A. Novelo. 1988. Plants and aquatic-flora of lake Pátzcuaro, Michoacán, Mexico. The Southwestern Naturalist 33:167-175.

Lot, A. 2000. Plantas acuáticas en los jardines botánicos: ideas sobre el desarrollo de la colecciones. Boletín Amaranto 13:13-25.

Lot, A. y A. Novelo. 2004. Iconografía y estudio de plantas acuáticas de la ciudad de México y sus alrededores. Instituto de Biología, UNAM/ Dirección de Divulgación Científica, UNAM. 206 p.

Lot, A., A. Novelo y P. Ramírez-García. 1998. Diversidad de la flora acuática mexicana.In T.P. Ramamoorthy, R. Bye, A. Lot y J. Fa (eds.), Diversidad biológica de México: orígenes y distribución. Instituto de Biología, UNAM, México, D.F. p. $563-580$.

Lot, A., A. Novelo, M. Olvera y P. Ramírez. 1999. Catálogo de angiospermas acuáticas de México. Hidrófitas estrictas emergentes, sumergidas y flotantes. Cuadernos del Instituto de Biología 33.Universidad Nacional Autónoma de México, México, D. F. 161 p.

Madrigal Guridi, X., A. Novelo Retana y A. Chacón Torres. 2004. Flora y vegetación acuáticas del lago de Zirahuén, Michoacán, México. Acta Botanica Méxicana 68: 1-38.
Manly, B. F. J. 2000. Multivariate statistical methods: a primer, segunda edición. Chapman and Hall/CRC, London. 215 p.

Pettit, N. E. y R. J. Naiman. 2007. Fire in the riparian zone: Characteristics and ecological consequences. Ecosystems 10: 673-687.

R Development Core Team. 2008. R: a language and environment for statistical computing. R Foundation for Statistical Computing, Vienna.URL: http://www.R-project.org; fecha de consulta: 14 enero, 2008.

Ramos Ventura, L. J. y A. Novelo Retana. 1993. Vegetación y flora acuáticas de la laguna de Yuriria, Guanajuato, México. Acta Botanica Mexicana 25:61-79.

Rodríguez y Guevara. 2000. Angiospermas: Catálogo de la biodiversidad en Michoacán. Secretaría de Desarrollo Urbano y Ecología, México, D. F. p. 103-152.

Rojas Moreno, J. y A. Novelo Retana. 1995. Flora y vegetación acuáticas del lago de Cuitzeo, Michoacán, México. Acta Botanica Mexicana 31: 1-17.

Romero López, B. E., J. L. León de la Luz, J. J. Pérez Navarro y G. de la Cruz Agüero. 2006. Estructura y composición de la vegetación de la barra costera El Mogote, Baja California Sur, México. Boletín de la Sociedad Botánica de México79: 21-32.

USDA. 2008. Plants database. United States Department of Agriculture. URL: http://plants.usda.gov/index.html; fecha de consulta: 19 enero, 2008.

Warners, D. P. 1989. Effects of controlled burning on a sedge meadow ecosystem in central Wisconsin. Tesis maestría, Universidad de Wisconsin, Madison, Wisconsin. 143 p.

Werner, K.J. y J.B. Zedler. 2002. How sedge meadow soils, microtopography, and vegetation respond to sedimentation. Wetlands 22:451-466.

Woo, I. y J.B. Zedler. 2002. Can nutrients aline shift a sedge meadow towards dominance by the invasive Typha $\mathrm{X}$ glauca? Wetlands 22:509-521.

Zar, J. H. 1999. Biostatistical analysis, 4a. edición. Prentice Hall. New Jersey. 663 p.

Zedler, J. B., J. C. Callaway, J. S. Desmond, G. Vivian-Smith, G. D. Williams, G. Sullivan, A. E. Brewster, B. K. Bradshaw. 1999. Californian salt-marsh vegetation: an improved model of spatial pattern. Ecosystems 2:19-35. 